\title{
Methods of Investigation of Equations that Describe Waves in Tubes with Elastic Walls and Application of the Theory of Reversible and Weak Dissipative Shocks
}

\author{
Igor Bakholdin ${ }^{1, \star}$ \\ ${ }^{1}$ Keldysh Institute of Applied Mathematics, Miusskaya sq. 4, 125047 Moscow, Russia
}

\begin{abstract}
Various models of a tube with elastic walls are investigated: with controlled pressure, filled with incompressible fluid, filled with compressible gas. The non-linear theory of hyperelasticity is applied. The walls of a tube are described with complete membrane model. It is proposed to use linear model of plate in order to take the bending resistance of walls into account. The walls of the tube were treated previously as inviscid and incompressible. Compressibility of material of walls and viscosity of material, either gas or liquid are considered. Equations are solved numerically. Three-layer time and space centered reversible numerical scheme and similar two-layer space reversible numerical scheme with approximation of time derivatives by Runge-Kutta method are used. A method of correction of numerical schemes by inclusion of terms with highorder derivatives is developed. Simplified hyperbolic equations are derived.
\end{abstract}

\section{Introduction}

Models that describe waves in elastic tubes are of great interest for technical and biological applications. Here equations based on the hyperelastic model and the complete membrane model are analyzed. Such equations were derived in [1] and analytically investigated in [2] and [3]. The purpose of this work is the generalization of the models, the application of the theory of reversible and weakly dissipative shocks [4], the development of numerical methods.

The equations of wave motion of the walls of an elastic incompressible cylindrical tube with fixed internal and external pressure are given by [2]

$$
\begin{gathered}
\left(R \sigma_{1} z^{\prime} / \lambda_{1}^{2}\right)^{\prime}-P^{*} r r^{\prime}=\rho R \ddot{z}, \quad\left(R \sigma_{1} r^{\prime} / \lambda_{1}^{2}\right)^{\prime}-\sigma_{2} / \lambda_{2}+P^{*} r z^{\prime}=\rho R \ddot{r}, \\
\lambda_{1}=\sqrt{r^{\prime 2}+z^{\prime 2}}, \quad \lambda_{2}=r / R, \quad \lambda_{3}=h / H, \quad \sigma_{i}=\lambda_{i} W_{\lambda_{i}}-p, \quad P^{*}=P / H .
\end{gathered}
$$

Here the prime denotes the derivative with respect to the variable $Z$, which stands for Lagrange initial longitudinal spatial coordinate, the upper dots denote derivative with respect to the time. Unknowns $z$ and $r$ determine the surface of the tube in the cylindrical system of coordinates, the $z$ axis of this system coincides with the central line of the tube. The parameter $P$ stands for the difference between internal and external pressures, the unknown $p$ is the pressure in the material. The parameter $\rho$ is the

\footnotetext{
^e-mail: ibbakh@yandex.ru
} 
density of material per square unit. The thickness of the wall is denoted as $h$. Here $\lambda_{i}$ are the principal stretches and $\sigma_{i}$ are the principal Cauchy stresses, $W$ is the stress function. It is assumed that in the absence of any external forces $z=Z, r=R, h=H$. Due to the condition of incompressibility,

$$
\lambda_{1} \lambda_{2} \lambda_{3}=1, \quad \sigma_{i}=\lambda_{i} \hat{W}_{i}, \quad \hat{W}_{i}=\hat{W}_{\lambda_{i}}, \quad \hat{W}=W\left(\lambda_{1}, \lambda_{2}, 1 /\left(\lambda_{1} \lambda_{2}\right)\right) .
$$

The stress function used in this paper for numerical calculations corresponds to the Gent material

$$
W=-\tilde{\mu} J_{m} \ln \left[1-\left(\lambda_{1}^{2}+\lambda_{2}^{2}+\lambda_{3}^{2}-3\right) / J_{m}\right] .
$$

It is considered that this stress function is applicable for rubber.

For the case of a fluid-filled tube with arbitrary pressure we must include the equations [3]

$$
\begin{aligned}
& \dot{r} z^{\prime}-r^{\prime} \dot{z}+v r^{\prime}+\frac{1}{2} r v^{\prime}=0, \\
& \rho_{f}\left(\dot{v} z^{\prime}-v^{\prime} \dot{z}+v v^{\prime}\right)+P^{\prime}=0 .
\end{aligned}
$$

Here $v$ is the fluid velocity, $\rho_{f}$ is the fluid density.

\section{Main generalizations and simplification of the model}

The analysis shows that for the case of the gas-filled tube the equation (2) must be replaced by

$$
\left(\dot{\rho_{f}} z^{\prime}-\rho_{f}^{\prime} \dot{z}\right) r^{2}+2 \rho_{f} r\left(\dot{r} z^{\prime}-r^{\prime} \dot{z}\right)+\left(\rho_{f} v r^{2}\right)^{\prime}=0 .
$$

In the barotropic case only one additional equation of state is required. An additional equation for energy is required for the complete gas dynamics model.

The viscosity of the fluid or gas may be taken into account if the additional term $c_{v 2} v^{\prime \prime}$ is included in (3). The viscosity of the material of the walls is enforced within the Kelvin-Voight model [5]

$$
\sigma_{i} \rightarrow \sigma_{i}+\sigma_{v i}, \quad \sigma_{v i}=v_{v i} \dot{\lambda}_{i}, \quad i=1,2,3 .
$$

It can be shown that for some simplified version of the equations this model leads to the inclusion of the term $c_{v 2} v^{\prime \prime}$ in the equation (3) also.

The compressibility of the material of the wall may be taken into account if a stress function (potential) without $p$ is used. A two dimensional potential (potential with hat) can be derived from the equation $\sigma_{3}=p_{e}$, where $p_{e}$ is the external pressure.

In order to take into account the meridional bending resistance of the thin walls additional "bending resistance pressure" must be formally included in (1) but not in (3) [6, 7]

$$
P \rightarrow P+P_{b}, \quad P_{b}=-\cos \alpha\left[\frac{\partial^{2}}{\partial x^{2}}\left(D \frac{\partial^{2}}{\partial x^{2}} r\right)-\tan \alpha \frac{\partial^{2}}{\partial x^{2}}\left(D \frac{\partial^{2}}{\partial x^{2}} z\right)\right], \quad \frac{\partial}{\partial x}=\frac{\cos \alpha}{z^{\prime}} \frac{\partial}{\partial Z}, \quad \tan \alpha=\frac{r^{\prime}}{z^{\prime}} .
$$

Here $\alpha$ is the angle of inclination of the wall of the tube. This approach is based on the GermainLagrange formula. The bending resistance coefficient $D$ must be calculated for the anisotropic linearized state

$$
D=\left\{W_{1}+\lambda_{1}\left(W_{11}-W_{13}\right)+\left[W_{3}+\lambda_{3}\left(W_{33}-W_{31}\right)\right] \lambda_{3} / \lambda_{1}\right\} h^{3} / 12 .
$$

If the geometric nonlinearity is not taken into account then $P_{b}=P_{b s}=-D r^{\prime \prime \prime \prime}$ and the bending pressure is included only in the equation for $\mathrm{r}$. Only the term $-b r^{\prime \prime \prime \prime}$ is added to the left part in this 
case. A problem is not well-posed if $\sigma_{1}<0$ and initial equations are used. The inclusion of the bending resistance enforces well-posedness.

A non-differential term related with the latitudinal (circular) bending resistance [8] may be included in pressure formula. But if the wall of the tube is weakly stretched (this is a typical case when inclusion of meridional bending resistance is required) or if $h / R$ is close to zero this term vanishes.

The term simplified equations is used in the theory of shocks to denote hyperbolic equations describing waves with slow variations. The simplified form of the controlled pressure equations is

$$
\dot{u}=q^{\prime} ; \quad \rho R \dot{q}=\left(R \sigma_{1} u / \lambda_{1}^{2}\right)^{\prime}-P^{*} r^{\prime}, \quad \lambda_{1}=u=z^{\prime}, \quad P^{*}=\sigma_{2} /\left(\lambda_{2} r u\right) .
$$

No simplification is required for the equations (2), (3), (4). These equations describe non-wave zones for Riemann problem solutions. Calculations of solutions of these equations require the use of special methods of calculations such as Godunov method in order to obtain generalized solutions with discontinuities.

\section{Numerical methods and results of calculations}

Standard three-layer time and space centered scheme and space centered two-layer perdictor-corrector schemes with approximation of time derivatives by Runge-Kutta method were used for all cases. These schemes were used for all models analysed in the theory of reversible and weakly dissipative shocks. The schemes are explicit usually. Similar implicit numerical schemes are used for the approximation of complex time-space derivatives if it is necessary.

For the case of fluid filled tube the equations were transformed to the form with excluded pressure

$$
\begin{gathered}
{\left[\rho_{f}^{*} z^{\prime}-\left(\frac{r^{\prime} \rho R^{2}}{r z^{\prime 2} \xi}\right)^{\prime}\right] \dot{v}-\left[\frac{r^{\prime} \rho R^{2}}{r z^{\prime 2} \xi}+\left(\frac{\rho R^{2}}{2 z^{\prime 2} \xi}\right)^{\prime}\right] \dot{v}^{\prime}-\frac{\rho R^{2}}{2 z^{\prime 2} \xi} \dot{v}^{\prime \prime}=\rho_{f}^{*}\left(v^{\prime} q-v v^{\prime}\right)+\left[-P_{b}^{*}+\frac{\mathcal{P}}{\xi}\right]^{\prime}+c_{v 2} v^{\prime \prime}-\left\{c_{v 4} v^{\prime \prime \prime \prime}\right\},} \\
\dot{r} z^{\prime}-r^{\prime} q+v r^{\prime}+\frac{1}{2} r v^{\prime}=0+\left\{c_{r 6} r^{\prime \prime \prime \prime \prime \prime \prime} z^{\prime}\right\}, \quad \dot{z}=q-\left\{c_{z} z^{\prime \prime \prime \prime}\right\}, \quad \rho R \dot{q}=\left(R \sigma_{1} \frac{z^{\prime}}{\lambda_{1}^{2}}\right)^{\prime}-\left(P^{*}+P_{b}^{*}\right) r r^{\prime}, \\
P^{*}+P_{b}^{*}=\frac{P+P_{b}}{H}=\frac{-1}{\xi}\left[\frac{r^{\prime} \rho R}{r z^{\prime 2}} \dot{v}+\frac{\rho R}{2 z^{\prime 2}} \dot{v}^{\prime}+\mathcal{P}\right], \quad \xi=1+\frac{r^{\prime 2}}{z^{\prime 2}}, \\
\mathcal{P}=\frac{1}{r z^{\prime}}\left[\left(R \sigma_{1} \frac{r^{\prime}}{\lambda_{1}^{2}}\right)^{\prime}-\frac{r^{\prime}}{z^{\prime}}\left(R \sigma_{1} \frac{z^{\prime}}{\lambda_{1}^{2}}\right)^{\prime}-\frac{\sigma_{2}}{\lambda_{2}}\right]-\frac{\rho R}{r z^{\prime 2}}(q-v)\left(\frac{z^{\prime} q-v r^{\prime}-\frac{1}{2} r v^{\prime}}{z^{\prime}}\right)^{\prime}-\frac{\rho R}{r z^{\prime 3}}\left(r^{\prime} q-v r^{\prime}-\frac{1}{2} r v^{\prime}\right)\left(\frac{1}{2} v^{\prime}-q^{\prime}\right) .
\end{gathered}
$$

Here terms in braces may be included for effectiveness of calculations (see below).

For the case of controlled pressure the three-layer scheme was mainly used [6]. But for cases of fluid-filled and gas-filled tubes boundary numerical instability is observed. For fluid-filled tubes this instability may be corrected by inclusion of dissipative zones near boundaries. An additional term with second-order derivative must be included in the equation for $v$. If the space step is decreased then the coefficient before this term must be increased. Good results are achieved if this term is approximated by the DuFort-Frankel method. This is not convenient for calculations. That is why two-layer predictor-corrector scheme was used for the cases of fluid-filled and gas-filled tube. This scheme possesses non-correct scheme dissipation. The growth of disturbances is observed for longtime calculations. But nevertheless convergence is achieved under a stability condition that is more sharper than the natural Courant condition. This effect may be removed if additional terms with higher-order derivatives are included. The magnitudes of the coefficients of this additional terms 
are taken to be proportional to the square of the space step. Hence the order of approximation of the scheme is preserved. Such approach may be used non only for the case of equations of tube. Estimations of the required magnitudes of the coefficients in the general case are made. Note that for Runge-Kutta method half of time step predictor is used. Growth of disturbances may be excluded if the predictor is applied for the entire time step. But such numerical scheme possesses great numerical dissipation and its application for non-dissipative and weakly dissipative problems is not convenient.

Numerical solutions of the Riemann problem are found. Results of calculations correspond to the theory of reversible and weakly dissipative shocks. Typical types of shock structures [4] are found for all cases. Examples of graphs for the case of controlled pressure are given in [6]. Here graphs of solutions for fluid-filled tube are presented in fig. 1, $v_{0}=0.1, R=1, \rho=1, \rho_{f}^{*}=1, \tilde{\mu}=2, J_{m}=30$, $z_{-\infty}^{\prime}=1.1, t=1000, b=0.1$. Here $v_{0}$ is initial velocity of fluid. Non-stationary chaotic structure of mainly longitudinal elastic waves moving to the left, solitary wave structure of mainly transversal elastic waves moving to the left, kink, solitary wave structure of mainly transversal elastic waves moving to the right and structure with emission of mainly longitudinal elastic waves moving to the right are noticed.

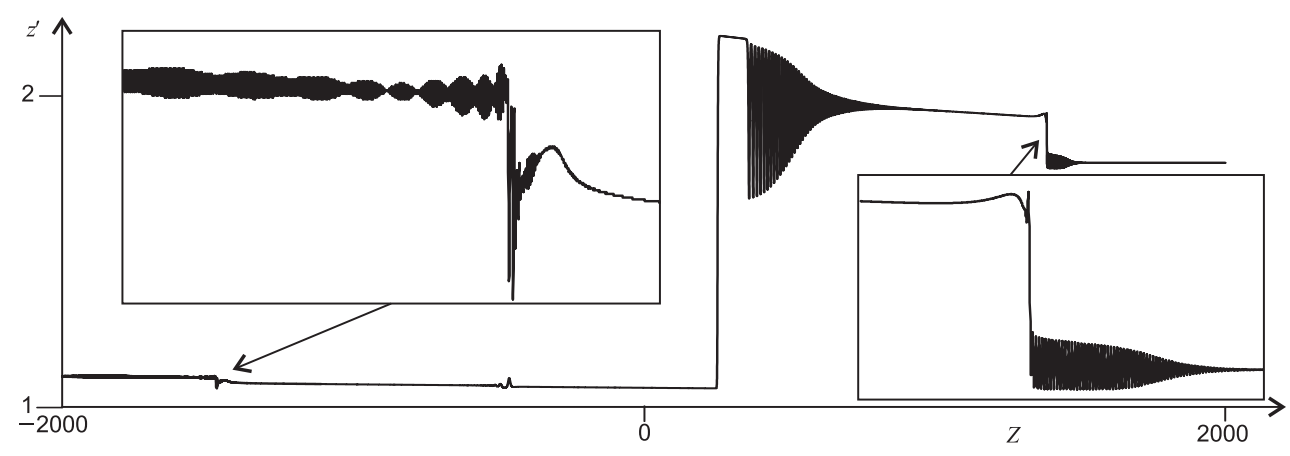

Figure 1. Solution of Riemann problem

The results of the investigation show that the theory of non-dissipative shocks is applicable for the equations of tubes with elastic walls. Effective numerical method of correction of the two-step scheme is developed. It can be used for reversible equations if the three-layer scheme is not applicable.

\section{Acknowledgement}

This work was supported by Russian Foundation for Basic Research, grant 15-01-04357a.

\section{References}

[1] M. Epstein and J. W. Johnston, Proc. Roy. Soc. London A 457, 2009, 1195-1213 (2001)

[2] Y. B. Fu and S. P. Pearce, IMA J. Appl. Math. 75, 4, 581-602 (2010)

[3] Y. B. Fu and A. Il'ichev, IMA J. Appl. Math. 75, 2, 257-268 (2010)

[4] I. B. Bakholdin, J. Appl. Math. Mech. 78, 6, 599-612 (2014)

[5] A. G. Kulikovskii and E. I. Sveshnikova, Nonlinear Waves in Elastic Media (CRC Press, Boca Raton, New York, London, Tokio, 1995)

[6] I. B. Bakholdin, J. Comp. Math. and Math. Phys. 55, 11, 1884-1898 (2015)

[7] I. B. Bakholdin, Prikl. Mat. Mekh. 81, 5, 593-609 (2017) (J. Appl. Math. Mech. in press)

[8] Y. B. Fu, J. L. Liu, and G. S. Francisco,J. of the Mechanics and Physics of Solids 90, 45-60 (2016) 